\title{
Validation of a retrospective respiratory gating method for small-animal CT scanners
}

\author{
Cristina Chavarrías, Student Member IEEE, Juan José Vaquero, Senior Member, IEEE, Alejandro Sisniega, Student \\ Member IEEE, Alexia Rodríguez-Ruano, María Luisa Soto-Montenegro, and Manuel Desco
}

\begin{abstract}
We propose a retrospective respiratory gating algorithm to generate dynamic CT studies. To this end, we compared three different methods of extracting the respiratory signal from the projections of small-animal cone-beam computed tomography (CBCT) scanners. Given a set of frames acquired from a certain axial angle, subtraction of their average image from each individual frame produces a set of difference images. The respiratory signals were extracted by analyzing the shape of the histogram of these difference images: we calculated the first four central and non-central moments. However, only odd-order moments produced the desired breathing signal, as the even-order moments lacked information about the phase. Each of these curves was compared to a reference signal recorded by means of a pneumatic pillow. Given the similar correlation coefficients yielded by all of them, we selected the mean to implement our retrospective protocol. Respiratory phase bins were separated, reconstructed independently and included in a dynamic sequence, suitable for cine playback. We validated our method in 5 adult rat studies by comparing profiles drawn across the diaphragm dome, with and without retrospective respiratory gating. Results showed a sharper transition in the gated reconstruction, with an average slope improvement of $60.7 \%$.
\end{abstract}

\section{INTRODUCTION}

$\mathrm{C}$ omputed tomography (CT) provides precise noninvasive morphological information for the diagnosis and treatment planning of many diseases. However, imaging the thorax and abdomen in vivo is limited by respiratory motion, which causes blurring and artifacts in the CT projections and in the reconstructed tomographic images. Prospective and retrospective methods have been proposed in the literature to compensate lung and rib motion. Retrospective algorithms [1]-[5] do not require any trigger signal during the acquisition, although they involve sorting the images into a different number of phase bins. Extensive research has been conducted to automatically extract the respiratory gating signal from the projection images before the data are reconstructed into three dimensions [1-3]. Most of the literature mentioned above focuses on human imaging, but both prospective [4],[ 5] and retrospective gating techniques [3] have also been applied to image small animals with dedicated CT scanners.

Manuscript received May 9, 2008. This project was supported by the CENIT Programme (Ministerio de Industria), CIBER CB07/09/0031 and RETICRECAVA (Ministerio de Sanidad y Consumo), and TEC2007-64731 / TCM (Ministerio de Educación y Ciencia).

All authors are with Laboratorio de Imagen Médica, Unidad de Medicina y Cirugía Experimental, Hospital General Universitario Gregorio Marañón, Madrid, Spain (telephone: +34-91-426-50-67, M. Desco email: desco@hggm.es)
In the present work, we validated a fast, retrospective algorithm which extracts the respiratory signal from CT projections in cone-beam geometry, thus avoiding the use of any additional respiratory monitoring instruments. The process is software-based and totally automatic.

\section{SIGNAL EXTRACTION}

From each set of frames acquired from the same axial angle, we obtained an average image which is then subtracted from each individual frame providing a set of difference images, $I_{d}$. In the area where there is lung movement, these difference images contain mainly positive values when they correspond to expiration and negative values for inspiration, while those pixels belonging to static areas have an almost zero value. A visual analysis of the histograms of these difference images indicates that both their mean value and symmetry change according to the respiratory phase (Fig. 1).

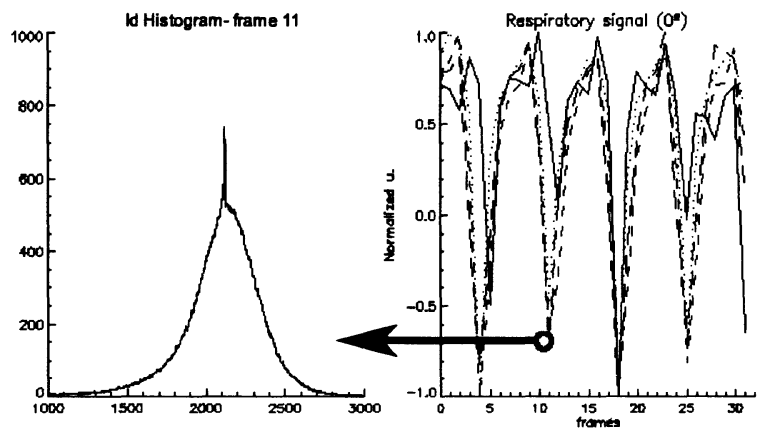

Fig. 1. Left: Histogram of one of the difference images, in this case at maximum inspiration (filtered by a boxcar of 10 pixels). Notice the peak corresponding to the static areas, that is, the grey pixels of Id. Right: the 3 respiratory signals extracted from the complete set of 32 frames: the dotted line corresponds to $\mu_{3}$, the third non-central moment, the dashed line represents $\gamma 3$ ', the skewness (normalized third central moment), long-dashed line represents the mean, $\mu_{1}$, and the continuous line represents the reference signal recorded with the pneumatic pillow.

We calculated the first four central and non-central moments of these difference images and normalized the resulting signals to between -1 and 1 . Even-order moments are always positive; therefore, the negative hemicycle of the respiratory signal becomes rectified to positive and is no longer representative of the subject's respiration. As $\mu_{1}$ ' is 
always zero, we finally tested the signals provided by $\mu_{1}, \mu_{3}$ (first and third non-central moments) and $\gamma_{3}$ ' (skewness).

The three curves corresponding to $\mu_{1}, \mu_{3}$ and $\gamma_{3}$ ' were compared to an external reference respiratory signal recorded during acquisition by means of a pneumatic pillow. The curve showing better correlation with the reference, $\mu_{1}$, was selected to represent the respiratory gating signal for our retrospective protocol.

In order to generate a dynamic study from our raw data, we divided the amplitude of the breathing cycle into four different bins, each one corresponding to a different respiratory phase. This approach was preferred over phase sorting, since it was previously demonstrated that it provides higher similarity between the frames assigned to each bin [6]. Averaging the frames assigned to each bin resulted in a set of four representative projections per angle. Finally, we reconstructed each bin independently, and obtained a dynamic study of the subject composed of four image volumes, one at end expiration, another at end inspiration, and two intermediate phases.

\section{VALIDATION AND RESULTS}

To carry out the validation we used the CT subsystem of an ARGUS PET/CT system (SUINSA Medical Systems)[7]. This system uses cone-beam geometry and a flat-panel detector. Acquisition was based on a $1^{\circ}$ step-and-shoot protocol covering $360^{\circ}$, with multi-frame setup to obtain 32 frames per gantry position at a speed of 8 frames/second. All the acquisitions were obtained with a pixel binning of four and the resulting image size was 512 × 512 pixels. X-ray source parameters were $50 \mathrm{kV}$ and $200 \mu \mathrm{A}$ and the total study duration was about $25 \mathrm{~min}$. The physiological signal used as a reference for validation was obtained during the acquisition by means of a pneumatic pillow placed under the subject's body connected to an air-pressure sensor. It was then amplified, digitalized, and recorded with a general-purpose laptop. We simultaneously extracted and recorded a synchronism signal from the scanner which indicated the periods of frame acquisition and the periods of gantry rotation, when no frames are acquired.

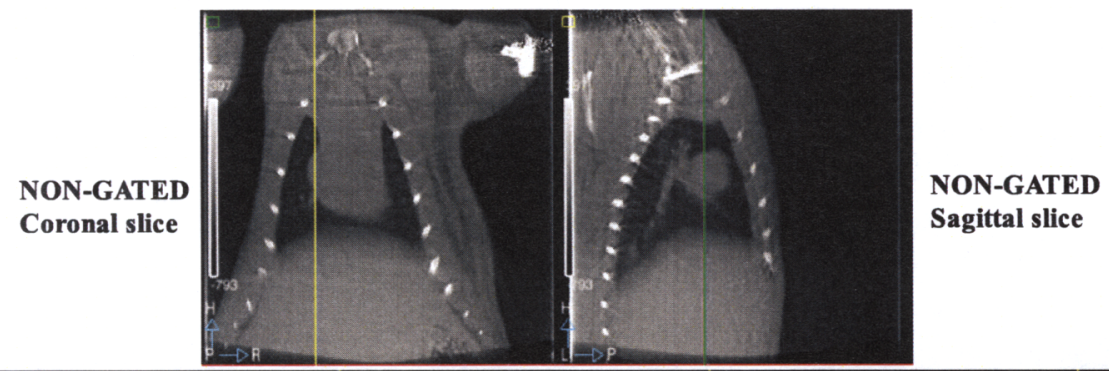

A)

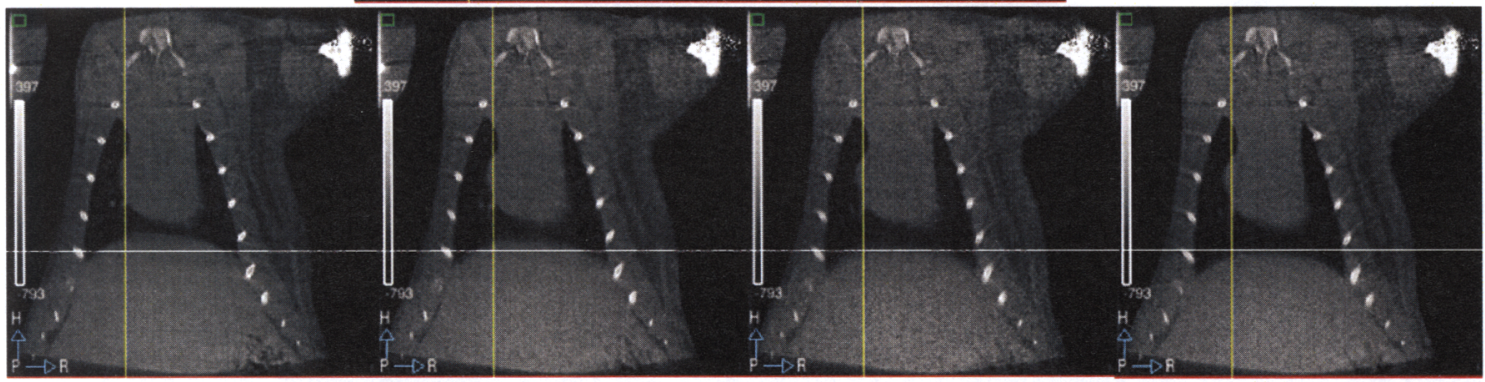

B)

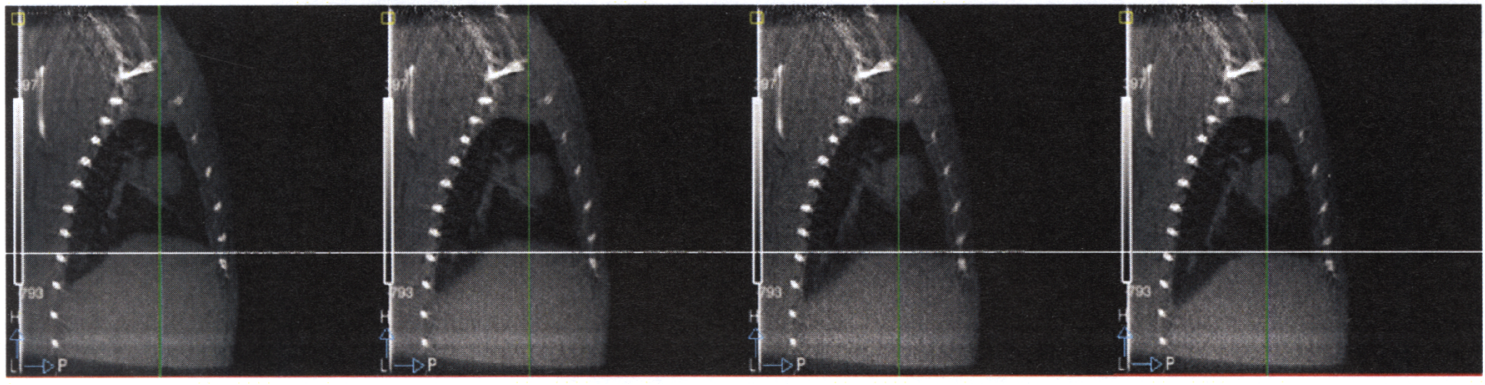

Fig. 2. Upper row: non-gated study. A) Coronal views of the resulting dynamic study and B) Sagittal views of the resulting dynamic study. The white lines drawn over the slices provide a common spatial reference to better appreciate the diaphragm motion. 
Both signals were recorded at $44 \mathrm{KHz}$ using an $\mathrm{A} / \mathrm{D}$ converter with USB interface (Edirol UA-1A, Roland Corporation), and resampled to $8 \mathrm{~Hz}$.

Once we calculated the three candidate signals in the proper time axis, we compared each of them with the reference signal recorded during acquisition by calculating their correlation coefficients. The signal selected for our retrospective protocol was the one with highest correlation coefficient, namely, the mean or first non-central moment.

Finally, after the binning process, all the 3D reconstructions were performed by means of a Feldkamp algorithm implemented in C. The dynamic studies obtained are shown in Fig. 2.

To assess the effectiveness our 4D-CT retrospective gating protocol we measured the sharpness of the diaphragm-lung transition in the reconstructed non-gated volume and in the gated image volume corresponding to bin 1 (end-expiration) of 5 Wistar rat studies. Three of them were anaesthetized with isoflurane and two with ketamine to account for different breathing patterns. We calculated the average slope from $10 \%$ to $90 \%$ height on 5 adjacent vertical profiles drawn at the diaphragm dome in coronal slices, following a procedure similar to that proposed by Moseley et al. [8]. Profile data were smoothed (10-pixel boxcar uniform filter) before measuring to reduce noise. An average improvement of $60.7 \%$ was achieved in the slope of gated reconstructions.

\section{CONCLUSION}

The breathing signal extracted from the projections with our method matched the expected pattern, as inferred from the visual inspection of the corresponding projections. The mean was the signal with higher correlation coefficient with the reference in all cases. Furthermore, the gating signals obtained from the mean, third non-central moment, and skewness curves were very similar to the reference signal, which supports the validity of either of them in a retrospective gating protocol. Reconstructed gated volumes at different phases showed a sharper diaphragm transition than that of the nongated studies. The comparison between profiles in these studies allowed us to quantify the improvement in resolution.

Direct extraction of the respiratory signal from the conebeam projections has an important advantage over external devices, since it not only avoids implementing the extra instrumentation, but it also makes the acquisition process much simpler and prevents skin contact artifacts. Furthermore, the proposed method is completely automatic, as it does not involve any user interaction (selecting regions of interest, thresholds, or landmarks in the lungs).

\section{REFERENCES}

1. Sonke J J, Zijp L, Remeijer P and van Herk M. Respiratory correlated cone beam CT. 2005 Med Phys. 32(4): p. 1176-86.

2. Rit $S$, Sarrut $D$ and Ginestet $C$. Respiratory signal extraction for $4 D C T$ imaging of the thorax from cone-beam CT projections. 2005 Med Image Comput Comput Assist Interv Int Conf. 8(Pt 1): p. 556-63.
3. Hu J, Haworth S T, Molthen R C and Dawson C A. Dynamic small animal lung imaging via a postacquisition respiratory gating technique using micro-cone beam computed tomography. 2004 Acad Radiol. 11(9): p. 961-70.

4. Ford N L, Nikolov H N, Norley C J, Thornton M M, Foster P J, Drangova $\mathrm{M}$ et al. Prospective respiratory-gated micro-CT of free breathing rodents. 2005 Med Phys. 32(9): p. 2888-98.

5. Badea C, Hedlund L W and Johnson G A. Micro-CT with respiratory and cardiac gating. 2004 Med Phys. 31(12): p. 3324-9.

6. Wink N, Panknin C and Solberg T D. Phase versus amplitude sorting of 4D-CT data. 2006 J Appl Clin Med Phys. 7(1): p. 77-85.

7. Vaquero J J, Redondo S, Lage E, Abella M, Sisniega A, Tapias G et al. Assessment of a New High-Performance Small-Animal X-ray Tomograph. 2008 IEEE T Nucl Sci. 55(3, part 1): p. 898-905.

8. Moseley D J, Hawkins M, Eccles C, Euler C, White E A, Bissonnette J et al. Respiratory Gated Cone-Beam CT Volumetric Imaging for External Beam Radiotherapy. 2005 Int J Radiat Oncol Biol Phys. 63(Supplement 1): p. S27-S28. 